\title{
Second Malignancies in Prostate Carcinoma Patients after Radiotherapy Compared with Surgery
}

\author{
David J. Brenner, D.sc. ${ }^{1}$ \\ Rochelle E. Curtis, M.A. ${ }^{2}$ \\ Eric J. Hall, D.sc. ${ }^{1}$ \\ Elaine Ron, Ph.D. ${ }^{2}$ \\ ${ }^{1}$ Center for Radiological Research, Columbia Uni- \\ versity, New York, New York. \\ ${ }^{2}$ Radiation Epidemiology Branch, National Cancer \\ ${ }^{2}$ Radiation Epidemiology Branch,
Institute, Bethesda, Maryland.
}

Supported in part by NIH Grant CA-530722.

The authors thank Dr. Tom Rohan for kindly providing data regarding smoking frequencies in prostate carcinoma patients. The authors also thank Lester Peters, Peter Schiff, Geoffrey Howe, and Carl Elliston for useful advice, and George Geise for computer support.

Address for reprints: David J. Brenner, D.Sc., Center for Radiological Research, Columbia University, 630 West 168th Street, New York, NY 10032.

Received April 14, 1999; revision received August 19, 1999; accepted September 2, 1999.
BACKGROUND. In the treatment of prostate carcinoma, radiotherapy and surgery are common choices of comparable efficacy; thus a realistic comparison of the potential long term sequelae, such as the risk of second malignancy, may be of relevance to treatment choice.

METHODS. Data regarding the rate of incidence from the Surveillance, Epidemiology, and End Results Program cancer registry (1973-1993) were used to compare directly second malignancy risks in 51,584 men with prostate carcinoma who received radiotherapy (3549 of whom developed second malignancies) with 70,539 men who underwent surgery without radiotherapy (5055 of whom developed second malignancies). Data were stratified by latency period, age at diagnosis, and site of the second malignancy. Directly comparing the risks in the radiotherapy group with those in the surgery group largely avoids problems associated with underreporting second malignancies.

RESULTS. Radiotherapy for prostate carcinoma was associated with a small, statistically significant increase in the risk of solid tumors $(6 \% ; P=0.02)$ relative to treatment with surgery. Among patients who survived for $\geq 5$ years, the increased relative risk reached $15 \%$, and was $34 \%$ for patients surviving $\geq 10$ years. The most significant contributors to the increased risk in the irradiated group were carcinomas of the bladder, rectum, and lung, and sarcomas within the treatment field. No significant increase in rates of leukemia was noted.

CONCLUSIONS. Radiotherapy for prostate carcinoma was associated with a statistically significant, although fairly small, enhancement in the risk of second solid tumors, particularly for long term survivors. The pattern of excess second malignancies among men treated with radiotherapy was consistent with radiobiologic principles in terms of site, dose, and latency. In absolute terms, the estimated risk of developing a radiation-associated second malignancy was 1 in 290 for all prostate carcinoma patients treated with radiotherapy, increasing to 1 in 70 for long term survivors ( $\geq 10$ years). Improvements in radiotherapeutic techniques, along with diagnosis at younger ages and earlier stages, are resulting in longer survival times for patients with prostate carcinoma. Because of the long latency period for radiation-induced tumors, this may result in radiation-related second malignancy risk becoming a more significant issue. Cancer 2000;88:398-406. (C) 2000 American Cancer Society.

KEYWORDS: second malignancies, radiation, prostate carcinoma, surgery.

$T^{1}$ he probability of a radiation-induced second malignancy after radiotherapy is a topic that has been widely discussed. ${ }^{1,2}$ In some situations in which radiotherapy clearly is the most efficacious treatment option, the issue is somewhat academic in that the benefits clearly will outweigh the risks. However, in other situations in which the choice of treatment modality is not clearcut, quantifying the risks of a radiation-induced second malignancy will allow one of the fac- 
tors of relevance in choosing between treatment options to be better defined.

Prostate carcinoma is the most common malignancy among men. In 1999, 179,300 new prostate carcinoma cases (comprising $29 \%$ of all new cancer cases among males) were expected to be diagnosed, and 37,000 prostate carcinoma deaths (comprising $13 \%$ of all cancer mortality in males) were expected in the U.S. ${ }^{3}$ Prostate carcinoma is one of the sites in which the choice of treatment between radiotherapy and surgery is least clearcut, ${ }^{4}$ and thus quantifying the relative risks of secondary malignancies for radiotherapy in comparison with surgery is of considerable societal interest.

Essentially, there are two approaches that can be taken practically to quantify the risks of radiationrelated second malignancies. A direct approach is to follow a group of patients treated at a single institution and compare the observed frequencies with an appropriate comparison group. ${ }^{5-7}$ In this situation, treatment protocols and possible subsequent retreatments generally can be reconstructed and analyzed reliably. Problems with this approach relate to the relatively small number of patients that can be studied and, often, to the absence of an appropriate comparison group. Studies using this approach also have the potential for selection bias associated with hospital referral patterns or protocol admittance criteria. The risks of a second malignancy after radiotherapy for prostate carcinoma have been evaluated in several single institution studies. ${ }^{6-8}$ Examination of the statistical power in these studies shows that even the largest such study (543 patients) had very limited power to detect and quantify realistic increases in rates of second malignancies after radiotherapy. ${ }^{7}$

An alternative approach involves retrospective studies based on data that are extracted from tumor registries. ${ }^{9-14}$ Using this approach, large numbers of individuals can be studied, yielding far greater statistical power, although there typically is less complete information available regarding treatment. In addition, tumor registry approaches can suffer from a lack of data regarding potential confounding factors, such as smoking or underreporting of secondary neoplasms. ${ }^{14}$ The methodology used in this article is designed to address this last issue by comparing risks in prostate carcinoma patients who received radiotherapy directly with prostate carcinoma patients who underwent surgery.

\section{MATERIALS AND METHODS}

Men with prostate carcinoma diagnosed between 1973-1993 were identified from the incidence files of nine cancer registries participating in the National
Cancer Institute's Surveillance, Epidemiology, and End Results (SEER) Program. ${ }^{15}$ The SEER Program is a set of geographically defined, population-based tumor registries, covering approximately $10 \%$ of the U.S. population.

SEER registry incidence files were searched for second primary malignancies (other than prostate carcinoma) that were diagnosed $\geq 2$ months after the initial prostate carcinoma diagnosis. For each patient, person-years at risk were accrued from 2 months after prostate carcinoma diagnosis until the date of death, date of last follow-up, date of diagnosis of subsequent primary tumor, or the end of 1993 , whichever came first. There were a total of 122,123 men in the database who had prostate carcinoma as their first primary tumor, who survived $\geq 2$ months after their initial diagnosis, and who received either radiation, with or without other treatments $(51,584$ men), or surgery without radiation (70,539 men) as their first course of therapy. Of the 51,584 men with prostate carcinoma who received radiotherapy, 3549 developed a second malignancy, and of the 70,539 men who underwent surgery, 5055 developed second malignancies.

Information regarding therapy given subsequent to the first course of therapy is not recorded in SEER. The probability of an individual who underwent prostate carcinoma surgery in 1990 receiving subsequent radiotherapy within 5 years was approximately $6 \% .{ }^{16}$

All male cancer sites recorded in the SEER database were analyzed separately; specifically, these were buccal, lip, tongue, salivary, gum and other oral sites, oropharynx, nasopharynx, hypopharynx, esophagus, stomach, small intestine, colon, rectum, liver or gallbladder, pancreas, nasal cavities, larynx, lung, breast, testis, kidney, bladder, melanoma, eye, brain or central nervous system, thyroid, endocrine, bone, connective tissue, non-Hodgkin lymphoma, Hodgkin disease, multiple myeloma, acute lymphocytic leukemia, chronic lymphocytic leukemia, acute nonlymphocytic leukemia, acute myelogenous leukemia, and chronic myelogenous leukemia.

The number of second malignancies expected, had these men experienced the same rates as the general population, was estimated based on sex specific, age specific, and calendar year specific incidence rates for all types of cancers combined (excluding prostate carcinoma) and for specific cancer sites; these incidence rates were applied to the appropriate number of person-years. Poisson regression methods for grouped survival data ${ }^{17}$ were used to estimate directly the percent increase in the relative risk of a second malignancy among prostate carcinoma patients treated with radiation compared with those treated with surgery (without radiotherapy). The data 
TABLE 1

Selected Characteristics of Men Diagnosed with Prostate Carcinoma, Initially Treated Either with Radiotherapy or with Surgery, as Reported to the SEER Program (1973-1993)

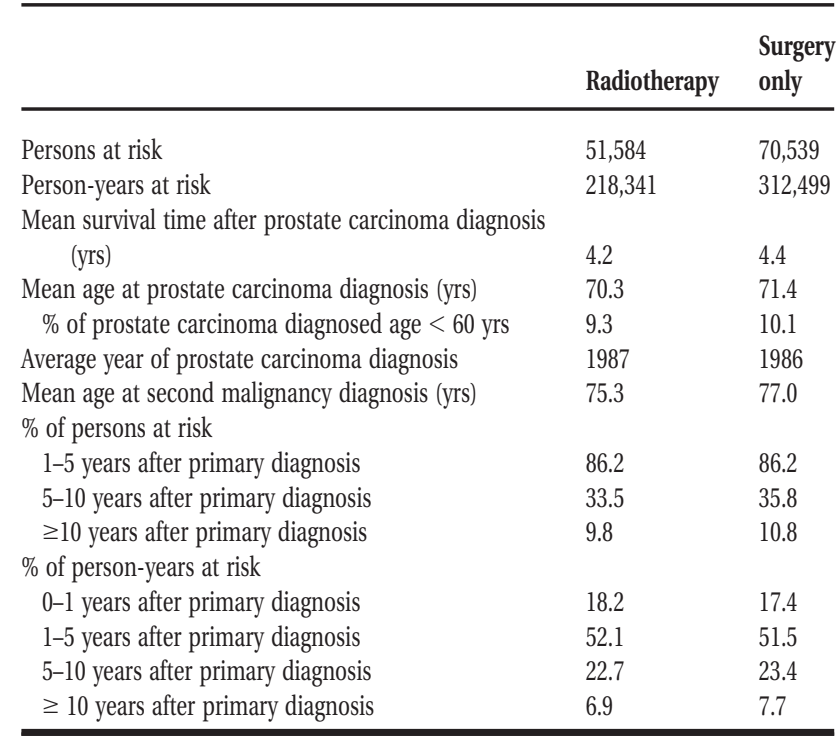

were stratified according to age at the time of diagnosis of prostate carcinoma $(<60,60-64,65-69,70-74$, $75-79,80-84$, and $85+$ years), and the time interval since prostate carcinoma diagnosis $(<5$ years, $\geq 5$ years, and $\geq 10$ years). 95\% confidence intervals (95\% $\mathrm{CI}$ ) and significance tests (two-sided) were estimated using standard likelihood ratio-based methods. ${ }^{17}$ This direct comparison of the risks observed among prostate carcinoma patients who received radiotherapy versus those who underwent surgery is preferable to comparisons with a general population because it avoids many of the problems associated with underreporting of second malignancies.

\section{RESULTS}

Table 1 shows selected characteristics of the 122,123 men with prostate carcinoma reported in the SEER database who were treated with radiotherapy or surgery. In terms of distributions of age, survival, and time at risk, the group of patients treated with radiation were remarkably similar to those undergoing surgery.

Because the SEER data come in part from the preprostate specific antigen (pre-PSA) era, the age distribution of the patients at the time of the primary prostate carcinoma diagnosis was somewhat older than the current age distribution of prostate carcinoma patients at diagnosis. For example, the average patient age at first diagnosis in the SEER database is 71 years compared with 69 years for prostate carcinoma patients in the National Cancer Data Base for $1995 .{ }^{18}$
In $1995,14.7 \%$ of prostate carcinoma patients were diagnosed at age $<60$ years compared with $9.7 \%$ in the SEER database.

Overall, 8604 men developed a subsequent malignancy (excluding prostate carcinoma), which was significantly less than the 9905 expected in the general population. Both the radiotherapy and the surgery treatment groups showed this apparent deficit of second malignancies when compared with the general population. However, when we restricted the analysis to subjects age $<60$ years at diagnosis there was no longer a deficit, a finding also reported by Kleinerman et al. ${ }^{19}$ For example, after restriction to ages at diagnosis $<60$ years, the standardized incidence ratio (observed/expected) for all malignancies at all times in the radiotherapy group was 1.05 compared with 0.89 when there was no age restriction. Therefore, it appears likely that the low standardized incidence ratio values both for radiotherapy and for surgery are a consequence of the comparatively elderly population under study. The current analysis largely overcame this underreporting problem by comparing the adjusted relative risks in the radiotherapy group directly with those in the surgery group.

Table 2 and Figure 1 show the percentage increase in the relative risk of developing a second malignancy after radiotherapy compared with surgery. There was little evidence of a difference in the risk for leukemia (or any lymphatic or hematopoietic malignancy) for patients treated with radiotherapy versus surgery, although the radiotherapy group did show a nonsignificant 5\% increased relative risk for leukemia during the first 5 years after diagnosis.

The solid tumors individually listed in Table 2 represent those for which there was either a significantly different relative risk for radiotherapy versus surgery, or for which there was a nonsignificant change in relative risk of $>20 \%$, for any time period. The risk of developing any second solid tumor at any time after diagnosis was significantly greater after radiotherapy than after surgery, by approximately $6 \%$ (95\% CI, $1-11 \%)$. As a function of time after diagnosis, this increased risk became greater, being 15\% (95\% CI, $6-24 \%$ ) after $\geq 5$ years, and $34 \%$ (95\% CI, $14-57 \%$ ) after $\geq 10$ years.

Of the specific solid tumor sites, both bladder carcinoma and lung carcinoma showed statistically significant radiation-associated increased relative risks at all times after diagnosis, and rectal carcinoma was increased significantly in the radiotherapy group for long term survivors. As illustrated in Figure 1 and Table 2, for those solid tumor sites in which a radiation-associated increase in risk was observed, radia- 
TABLE 2

Comparison of Risks of Developing Second Malignancies for Prostate Carcinoma Patients Treated with Radiotherapy versus Surgery Only, As a Function of Time after Diagnosis

\begin{tabular}{|c|c|c|c|c|c|c|c|c|c|}
\hline \multirow[b]{3}{*}{ Second malignancy ${ }^{\mathrm{a}}$} & \multirow{2}{*}{\multicolumn{3}{|c|}{ Radiotherapy }} & \multirow{2}{*}{\multicolumn{3}{|c|}{ Surgery }} & \multicolumn{3}{|c|}{ Radiotherapy vs. surgery } \\
\hline & & & & & & & \multirow{2}{*}{$\begin{array}{l}\% \text { increase in risk: } \\
\text { RT vs. surgery }\end{array}$} & \multirow{2}{*}{$\begin{array}{l}95 \% \mathrm{CI} \text { of } \% \\
\text { increase in risk }\end{array}$} & \multirow[b]{2}{*}{$P$ value } \\
\hline & Observed & Expected & $(0 / E)_{\mathrm{RT}}$ & Observed & Expected & $(0 / E)_{\text {surgery }}$ & & & \\
\hline All second malignancies ${ }^{\mathrm{c}}$ (all yrs) & 3549 & 3991 & 0.89 & 5055 & 5914 & 0.86 & 4 & {$[-1,9]$} & 0.08 \\
\hline$\geq 5$ yrs & 1185 & 1285 & 0.92 & 1646 & 2008 & 0.82 & 11 & {$[3,20]$} & 0.007 \\
\hline$\geq 10 \mathrm{yrs}$ & 305 & 318 & 0.96 & 393 & 528 & 0.75 & 27 & {$[9,48]$} & 0.002 \\
\hline All solid tumors ${ }^{\mathrm{d}}$ (all yrs) & 3171 & 3589 & 0.88 & 4441 & 5305 & 0.84 & 6 & {$[1,11]$} & 0.02 \\
\hline$\geq 5$ yrs & 1065 & 1152 & 0.92 & 1432 & 1797 & 0.80 & 15 & {$[6,24]$} & 0.0009 \\
\hline$\geq 10 \mathrm{yrs}$ & 280 & 284 & 0.99 & 344 & 471 & 0.73 & 34 & {$[14,57]$} & 0.0004 \\
\hline Bladder (all yrs) & 455 & 414 & 1.10 & 608 & 628 & 0.97 & 15 & {$[2,31]$} & 0.02 \\
\hline$\geq 5$ yrs & 164 & 137 & 1.20 & 168 & 219 & 0.77 & 55 & {$[24,92]$} & 0.0001 \\
\hline$\geq 10 \mathrm{yrs}$ & 46 & 35 & 1.32 & 44 & 59 & 0.75 & 77 & {$[14,163]$} & 0.01 \\
\hline Rectum (all yrs) & 198 & 242 & 0.82 & 298 & 363 & 0.82 & -2 & {$[-18,18]$} & 0.87 \\
\hline$\geq 5$ yrs & 73 & 77 & 0.95 & 86 & 121 & 0.71 & 35 & {$[-1,86]$} & 0.06 \\
\hline$\geq 10 \mathrm{yrs}$ & 22 & 19 & 1.18 & 17 & 31 & 0.55 & 105 & {$[9,292]$} & 0.03 \\
\hline Colon (all yrs) & 541 & 584 & 0.93 & 823 & 903 & 0.91 & 0 & {$[-10,12]$} & 0.97 \\
\hline$\geq 5$ yrs & 178 & 196 & 0.91 & 266 & 317 & 0.84 & 7 & {$[-11,30]$} & 0.47 \\
\hline$\geq 10 \mathrm{yrs}$ & 45 & 50 & 0.91 & 63 & 85 & 0.74 & 24 & {$[-16,81]$} & 0.29 \\
\hline Lung (all yrs) & 845 & 1050 & 0.80 & 1087 & 1485 & 0.73 & 11 & {$[1,21]$} & 0.03 \\
\hline$\geq 5 \mathrm{yrs}$ & 302 & 328 & 0.92 & 369 & 491 & 0.75 & 22 & {$[5,42]$} & 0.01 \\
\hline$\geq 10 \mathrm{yrs}$ & 79 & 79 & 1.01 & 88 & 126 & 0.70 & 42 & {$[5,93]$} & 0.02 \\
\hline Sarcomas in field (all yrs) & 38 & 21 & 1.80 & 32 & 31.4 & 1.02 & 85 & {$[15,201]$} & 0.01 \\
\hline$\geq 5$ yrs & 17 & 6.8 & 2.50 & 11 & 10.7 & 1.03 & 145 & {$[15,444]$} & 0.02 \\
\hline$\geq 10 \mathrm{yrs}$ & 5 & 1.7 & 2.91 & 3 & 2.9 & 1.05 & 217 & {$[-23,1461]$} & 0.11 \\
\hline Distant sarcomas (all yrs) & 31 & 22 & 1.40 & 32 & 33.2 & 0.97 & 51 & {$[-9,152]$} & 0.11 \\
\hline$\geq 5$ yrs & 10 & 7.2 & 1.39 & 11 & 11.5 & 0.96 & 36 & {$[-44,225]$} & 0.49 \\
\hline$\geq 10 \mathrm{yrs}$ & 2 & 1.9 & 1.08 & 1 & 3.1 & 0.32 & 251 & {$[-67,7584]$} & 0.29 \\
\hline Leukemia $^{\mathrm{e}}$ (all yrs) & 96 & 92 & 1.04 & 146 & 146 & 1.00 & 0 & {$[-23,30]$} & 0.98 \\
\hline $0-5 \mathrm{yrs}^{\mathrm{f}}$ & 67 & 62 & 1.09 & 95 & 95 & 1.00 & 5 & {$[-24,44]$} & 0.78 \\
\hline$\geq 5 \mathrm{yrs}^{\mathrm{f}}$ & 29 & 31 & 0.94 & 51 & 50 & 1.01 & -8 & {$[-43,45]$} & 0.73 \\
\hline
\end{tabular}

0: observed; E: expected; RT: radiotherapy; 95\% CI: 95\% confidence interval.

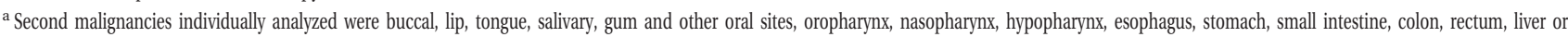
gallbladder, pancreas, nasal cavities, larynx, lung, breast, testis, kidney, bladder, melanoma, eye, brain or central nervous system, thyroid, endochrine, bone, connective tissue, non-Hodgkin lymphoma, Hodgkin disease, multiple myeloma, acute lymphocytic leukemia, chronic lymphocytic leukemia, acute nonlymphocytic leukemia, acute myelogenous leukemia, and chronic myelogenous leukemia. For brevity, data are shown only for those sites for which there was either a significantly increased relative risk for radiotherapy versus surgery (in either direction), or for which there was a nonsignificant increased relative risk of $>$ $20 \%$ for any time period.

${ }^{\mathrm{b}}$ Percent increase in relative risk for radiotherapy (RT) versus surgery (100 [1-RR $\left.\left.\mathrm{RT}_{\mathrm{RT}} / \mathrm{RR}_{\text {surgery }}\right]\right)$, in which the relative risks (RR) are calculated using Poisson models adjusted for age at prostate carcinoma diagnosis and time since prostate carcinoma diagnosis.

${ }^{\mathrm{c}}$ All malignancies listed in Footnote a, combined.

${ }^{\mathrm{d}}$ All malignancies listed in Footnote a, excluding leukemias and lymphomas, combined.

excluding chronic lymphocytic leukemia.

${ }^{\mathrm{f}}$ Note different time periods for leukemia.

tion-associated risk increased with increasing survival time.

The risk of developing a sarcoma within the treatment field was significantly larger after radiotherapy compared with surgery. The increase in risk was $85 \%$ (95\% CI, 15-201\%) at all times after diagnosis, reaching $145 \%$ (95\% CI, $15-444 \%)$ at $\geq 5$ years after diagnosis; the increased risk at $\geq 10$ years was no longer statistically significant, most likely due to the small number of cases. The risk of sarcomas outside the treatment field did not differ significantly between the two treatment groups, and for the majority of follow-up intervals generally was significantly lower than the risk for sarcomas within the treatment field.

Table 3 provides estimates of the absolute numbers of second solid tumors that could be attributed to the radiotherapy treatment. For example, among the 17,327 men who underwent radiotherapy and survived $>5$ years, there were an estimated 139 extra solid tumors, corresponding to 1 per 125 men, or 1 per 465 person-years at risk. The significant contributors to these extra solid tumors were bladder (estimated 58 

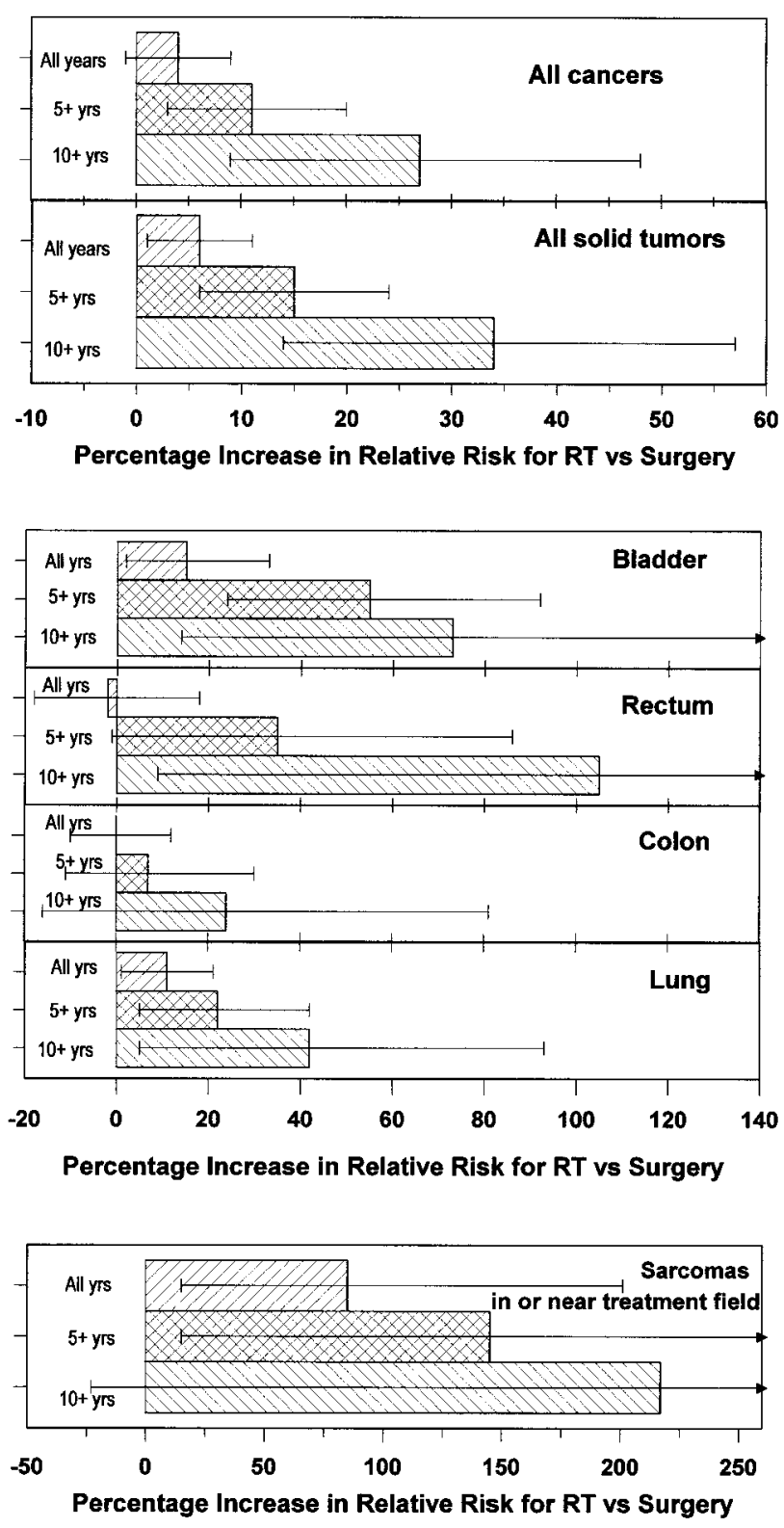

FIGURE 1. Percentage increase in relative risks for various second tumors in prostate carcinoma patients who received radiotherapy (RT), relative to the risks in those who underwent surgery without RT. Results are adjusted for age at time of prostate carcinoma diagnosis and time since prostate carcinoma diagnosis. Bars represent $95 \%$ confidence limits. Note the different scales for each panel.

tumors among the 17,327 men), lung (54 tumors), rectum (19 tumors), and sarcomas in the radiation field (9 tumors).

\section{DISCUSSION}

Because of the large numbers of cases involved in this study, comparisons between the risks in the radiation and surgery groups could be made with significant statistical power for all sites combined, for individual sites, and for different follow-up intervals. This approach is preferable to comparisons with a general population because it avoids many of the problems associated with underreporting of second malignancies.

Specifically, both the radiotherapy and the surgery treatment groups showed an apparent deficit of second malignancies when compared with the general population. Based on the results when analysis was restricted to those age $<60$ years at diagnosis (in which there was no longer a deficit of second malignancies), it appears likely that the apparent deficits both for radiotherapy and for surgery are a consequence of underreporting second tumors in the comparatively elderly population under study. This conclusion, also discussed by Kleinerman et al., ${ }^{19}$ suggests that estimating second tumor risk by comparison with a matched healthy population may lead to misleading results, particularly when the first tumors are diagnosed predominantly in elderly populations. The current analysis largely overcomes this underreporting problem by directly comparing the adjusted risks in the radiotherapy and the surgery groups, an approach that also is feasible, for example, for breast carcinoma. $^{5}$

\section{Solid Tumors}

Overall, there was a small but significant increase in solid tumors in the radiotherapy group compared with the surgery only group. There also was a clear pattern of increasing risk of solid tumors with increasing survival time ( $\geq 5$ years and $\geq 10$ years) in patients treated with radiotherapy compared with those undergoing surgery. Previous studies of the latency period of radiation-induced solid tumors suggest an average latency period of $5-15$ years, ${ }^{20}$ and the same temporal pattern in the current study lends support to the notion that the increased risks in the radiation group are linked causally to the radiation exposure.

\section{Leukemia}

There was no overall difference in the leukemia risk between the radiotherapy and the surgery groups. Based on the experience of the atomic bomb survivors, any radiation-related excess of leukemias would be expected to occur largely within the first 5 years after exposure ${ }^{21}$ and although a small (5\%, not statistically significant) increase in leukemia risk was observed within 5 years of diagnosis, the leukemia risks are lower than those projected from atomic bomb survivors. ${ }^{21}$ Lower leukemia risks than those projected from the atomic bomb survivors also were reported in 
TABLE 3

Estimated Absolute Numbers of Second Solid Tumors in the Radiotherapy Group Associated with Radiotherapy Treatment

\begin{tabular}{|c|c|c|c|c|c|}
\hline & Persons at risk & $\begin{array}{l}\text { Person-years } \\
\text { at risk }\end{array}$ & $\begin{array}{l}\text { Estimated no. of } \\
\text { solid tumors } \\
\text { associated with RT }\end{array}$ & $\begin{array}{l}\text { Estimated RT-associated } \\
\text { solid tumors/person at } \\
\text { risk }\end{array}$ & $\begin{array}{l}\text { Estimated RT-associated } \\
\text { solid tumors/person- } \\
\text { years at risk }\end{array}$ \\
\hline All years & 51,584 & 218,341 & 179 & 1 per 290 & 1 per $1220 \mathrm{PY}$ \\
\hline$\geq 5$ years after diagnosis & 17,327 & 64,700 & 139 & 1 per 125 & 1 per 465 PY \\
\hline$\geq 10$ years after diagnosis & 5046 & 15,053 & 71 & 1 per 70 & 1 per 212 PY \\
\hline
\end{tabular}

RT: radiotherapy; PY: person-years.

women who received radiotherapy for cervical or endometrial carcinoma, ${ }^{9,10}$ and it is plausible that the differences noted when compared with the atomic bomb survivor data are a consequence of the much higher dose to the bone marrow in the radiotherapeutic situation, causing cell death in potentially oncogenic damaged cells. ${ }^{10,22,23}$ However, conclusions regarding leukemia risks must be viewed in light of the limited statistical power available in the current study because leukemia is a comparatively rare malignancy.

\section{Individual Sites}

Analyzing the solid tumors site by site, there were significant radiation-associated increases in bladder carcinoma (consistent with the findings of Neugut et al. ${ }^{12}$ and Pawlish et al. ${ }^{13}$ ), rectal carcinoma, and lung carcinoma, as well as sarcoma in or near the treatment field. As with the overall data, there is a strong indication of an increasing radiation-associated risk with increasing survival time for the individual sites.

Although the largest number of radiation-associated malignancies clearly are carcinomas, the largest increase in relative risk is for in-field sarcomas, although the increase in out-of-field sarcomas was not statistically significant. In the majority of other studies radiation-induced sarcomas occur only in heavily irradiated sites, close to the treatment volume. ${ }^{24-27}$ These observations most likely reflect a different mechanism for the induction of sarcomas compared with carcinomas; the target cells for sarcomas typically are dormant cells and large doses are needed to produce sufficient tissue damage to stimulate cellular proliferation..$^{28}$ The sarcoma data in this study appear to follow this pattern, with significant radiation-associated risks being observed for sites in and close to the treatment volume but not for more distant sites, which received lower doses.

\section{Lung Carcinoma}

It is interesting to note that the radiation-associated risk for carcinoma of the lung, which is relatively distant from the prostate treatment volume, is elevated significantly and not that different from those for carcinomas of the bladder and rectum. Due to the treatment techniques used over the majority of the time covered by the current study (before around 1993, typically whole pelvic ${ }^{60} \mathrm{Co}$ irradiation ${ }^{29}$ ), the dose to the lung was around 0.6 Gray (Gy), ${ }^{29,30}$ nearly 2 orders of magnitude less than the doses to the bladder and rectum. The approximate constancy of the carcinoma risk over such a wide range of doses may reflect the fact that carcinomas, originating in actively dividing or hormonally controlled cells, can be induced efficiently by relatively low doses of radiation, as evidenced by the atomic bomb survivor data, ${ }^{20}$ but the carcinoma risk at high doses is attenuated by the effects of cell killing. ${ }^{23}$ Overall, this can produce a relatively flat dose-response relation for carcinoma induction over a wide dose range, which could explain why some lightly irradiated sites exhibit carcinoma risks similar to those in sites that received much higher doses.

There is a second factor, unrelated to radiation exposure, that might have contributed to the excess risk in the lung, namely, that a smoking history often is considered a counterindication for surgery, representing a high preoperative risk factor. ${ }^{31}$ Thus a higher proportion of smokers (and therefore lung tumors) might be expected in the radiation group. To investigate this possible bias, we analyzed the results of an earlier case-control study on smoking habits in Canadian prostate carcinoma patients. ${ }^{32}$ The results are shown in Table 4. Of 408 prostate carcinoma patients in the study, smoking and treatment information were available for 393, 283 of whom received either surgery without radiotherapy or radiotherapy with or without other treatments. In the surgery group, $16 \%$ were current smokers and $79 \%$ ever smokers, compared with $15 \%$ and $80 \%$, respectively, in the radiotherapy group. These results suggest that the increased lung carcinoma rates that were observed in the SEER radiotherapy group may not be the result of smoking-related biases.

A further indication that the increases in lung carcinoma rates in the radiotherapy group may indeed 
TABLE 4

Proportions of Smokers among Prostate Carcinoma Patients Undergoing Either Surgery without Radiotherapy or Radiotherapy with or without 0 ther Treatments ${ }^{\mathrm{a}}$

\begin{tabular}{lll}
\hline & $\begin{array}{l}\text { Proportion of } \\
\text { ever smokers }\end{array}$ & $\begin{array}{l}\text { Proportion of } \\
\text { current smokers }\end{array}$ \\
\hline Surgery without radiotherapy & 0.79 & 0.16 \\
Radiotherapy & $(79 / 94)$ & $(15 / 94)$ \\
& 0.80 & 0.15 \\
& $(151 / 189)$ & $(29 / 189)$ \\
\hline
\end{tabular}

${ }^{a}$ Data derived from results of a case-control study reported by Rohan et al. ${ }^{32}$

be related to radiation comes from examining the time course of the increased risk. In fact, the temporal pattern of the increased lung carcinoma risk in the radiation group is characteristic of solid tumors induced by radiation exposure. ${ }^{20}$ From $0-5$ years the increase in relative risk for the radiotherapy versus the surgery group was $5 \%$, increasing to $16 \%$ for $5-10$ years and to $42 \%$ for $\geq 10$ years, a temporal pattern that would be unlikely to be attributable to smoking.

\section{Comparison with Second Malignancy Rates after Radiotherapy for Cervical Carcinoma}

It is of interest to compare the results of the current study on prostate carcinoma patients with those of the corresponding tumor registry-based cohort studies of second tumors in cervical carcinoma patients. ${ }^{9,33}$ Such comparisons must of course be assessed in light of the different temporal characteristics of the two tumors; the average age at diagnosis for invasive cervical carcinoma (in the SEER database) is approximately 50 years compared with approximately 70 years for prostate carcinoma. This allows considerably longer follow-up times in the cervical carcinoma study (average follow-up, approximately 10 years $^{9}$ ) compared with the current study (average follow-up, approximately 4 years).

In fact the average radiation doses to various organs for the treatment of the two sites in the 1970s and 1980s were not dissimilar. For example, for the kidney and the lung, the estimated average doses associated with cervical carcinoma radiotherapy were approximately 2 Gy and $0.3 \mathrm{~Gy}$, respectively, ${ }^{9,33}$ whereas the corresponding average doses for prostate carcinoma radiotherapy were approximately 2 Gy and 0.6 Gy, respectively. ${ }^{29,30}$ In general, the doses from the two treatments to any given organ were within about a factor of 2 of each other.

Compared with prostate carcinoma, a much lower percentage of patients with invasive cervical carcinoma do not receive radiotherapy. A recent update of the cervical carcinoma tumor registry cohort study ${ }^{9,33}$ analyzed data for 49,900 women with invasive cervical carcinoma who underwent radiotherapy and 16,700 who received no radiotherapy compared with 51,600 and 70,500 men, respectively, in the current prostate carcinoma study (Table 1). This limits the power of direct comparisons between cervical carcinoma patients who did or did not receive radiotherapy, and indeed in their cohort studies Boice et al. reported radiation risks in cervical carcinoma patients relative to the general population, rather than relative to nonirradiated cervical carcinoma patients. ${ }^{9,33}$ As discussed earlier, comparisons with the general population are more prone to biases and confounding effects, such as smoking, misdiagnosis, and underreporting. However, comparisons between a radiotherapy group and a nonradiotherapy group still are practical for cervical carcinoma patients when disease sites are combined. For example, from the data for patients with invasive cervical carcinoma reported by Kleinerman et al., ${ }^{9}$ the percentage increase in relative risk for all solid tumors in the radiotherapy group relative to the nonradiotherapy group was $12 \%(95 \% \mathrm{CI}, 4-20 \%)$, increasing to $15 \%$ (95\% CI, 5-26\%) for $\geq 10$ year survivors and to $26 \%$ (95\% CI, $9-47 \%$ ) for $\geq 20$ year survivors; these increased risks may be compared with the results in Table 2 for prostate carcinoma patients of $6 \%(95 \% \mathrm{CI}, 1-11 \%)$, increasing to $34 \%$ (95\% CI, $14-57 \%$ ) for $\geq 10$-year survivors. As observed in the current study on prostate carcinoma patients, for the patients with invasive cervical carcinoma there was a nonsignificant increase in the early leukemia risk in the radiotherapy group relative to the nonradiotherapy group. ${ }^{9}$

For patients with cervical carcinoma the lung is one of the five individual sites for which a statistically increased second malignancy was observed in the radiotherapy group relative to the nonradiotherapy group $^{9}$ (increased risk 38\%; 95\% CI, $17-65 \%$; $P=$ 0.0001). This again supports the notion that the increased risk of lung carcinoma observed in the current study is related to radiation exposure.

Several caveats are appropriate in assessing these comparisons between patients with invasive cervical carcinoma who did or did not undergo radiotherapy. ${ }^{9,33}$ First, to our knowledge tests of homogeneity between the radiotherapy and nonradiotherapy groups with regard to smoking patterns are not available for cervical carcinoma patients (see Table 4 for such comparisons for the current prostate carcinoma study). Second, women with invasive cervical carcinoma who underwent radiotherapy typically were older than those who did not, ${ }^{33}$ although the average follow-up time in both groups was approximately the 
same. ${ }^{9,33}$ Third, the Danish tumor registry, which is the largest contributor to the cervical carcinoma study, ${ }^{9}$ has reported significant misclassification problems, specifically that up to $24 \%$ of individuals in the "nonradiotherapy" group actually may have undergone radiotherapy; ${ }^{34}$ this effect would lead to an underestimation of the radiation-associated risk. Bearing these caveats in mind, the results for invasive cervical carcinoma patients are reasonably consistent with the current results for prostate carcinoma patients.

\section{Relevance to Current Radiotherapy Treatment Techniques for Prostate Carcinoma}

In the current study, the mean treatment year for the radiotherapy group was 1987 . Despite the recent trend toward higher prescribed doses for prostate carcinoma radiotherapy, the adoption of smaller field, higher energy, radiotherapeutic treatments has resulted in significantly lower doses to distant organs compared with those from earlier, larger field, ${ }^{60} \mathrm{Co}$ treatments. ${ }^{35}$ By contrast, external beam radiotherapy treatment for prostate carcinoma typically is now performed using nominal photon energies $>18 \mathrm{MeV},{ }^{36}$ potentially producing a significant and highly effective photoneutron dose (approximately 0.2 Sievert) to distant organs. ${ }^{37}$

\section{Significance for Treatment Choice}

Radiotherapy for prostate carcinoma was associated with an overall small increase $(4 \% ; P=0.08)$ in the risk of all second malignancies relative to those patients treated with surgery, and with a significantly elevated risk of second solid tumors (6\%; $P=0.02)$. This overall enhanced risk for solid tumors is larger for long term survivors, increasing to $15 \%(P<0.001)$ after $\geq 5$ years, and to $34 \%(P<0.001)$ after $\geq 10$ years. Although the increased risks of solid tumors in the radiotherapy group are significant, the absolute risks still are fairly small; our best estimate is that the risk of developing a radiation-associated second malignancy after radiotherapy for prostate carcinoma is 1 in 290 (all years), 1 in 125 for $\geq 5$-year survivors, and 1 in 70 for $\geq 10$-year survivors.

Because the estimated absolute risks of radiationassociated second malignancies are fairly small, they might appear unlikely to play a significant role in choosing between treatment options. However, the trend toward prostate carcinoma diagnosis at a younger age and at an earlier stage ${ }^{18}$ as well as recent improvements in radiotherapeutic techniques, ${ }^{38,39}$ suggest that survival times after radiotherapy for prostate carcinoma will increase. In light of the long (5-15 years) average latency period for radiation-induced solid tumors, ${ }^{20}$ increased survival times would be ex- pected to result in increasing radiation-induced second tumor rates, and thus radiation-related second tumor risk could, in the future, become a more significant issue in making treatment decisions.

\section{REFERENCES}

1. Curtis RE. Second cancers following radiotherapy for cancer. In: Boice JD Jr., editor. Implications of new data on radiation cancer risk. Proceedings of the 32nd Annual Meeting of the National Council on Radiation Protection and Measurements. Bethesda: NCRP Publications; 1997:79-94.

2. Boice JD Jr, Travis LB. Body wars: effect of friendly fire. J Natl Cancer Inst 1995;87:705-6.

3. Landis SH, Murray T, Bolden S, Wingo PA. Cancer statistics, 1999. CA Cancer J Clin 1999;49:8-31.

4. Brenner DJ, Hall EJ. Making the radiotherapy decision. Los Angeles: Lowell Press, 1996.

5. Zucali R, Luini A, Del Vecchio M, Sacchini V, Sverzellati E, Stucchi C, et al. Contralateral breast cancer after limited surgery plus radiotherapy of early mammary tumors. Eur J Surg Oncol 1987;13:413-7.

6. Johnstone PA, Powell CR, Riffenburgh R, Rohde DC, Kane CJ. Second primary malignancies in T1-3N0 prostate cancer patients treated with radiation therapy with 10-year followup. J Urol 1998;159:946-9.

7. Movsas B, Hanlon AL, Pinover W, Hanks GE. Is there an increased risk of second primaries following prostate irradiation? Int J Radiat Oncol Biol Phys 1998;41:251-5.

8. Liskow AS, Romas N, Ozzello L, Suarez R, Veenema R, Chang $\mathrm{CH}$. Multiple primary tumors in association with prostatic cancer. Cancer 1984;54:2549-55.

9. Kleinerman RA, Boice JD Jr., Storm HH, Sparen P, Andersen A, Pukkala E, et al. Second primary cancer after treatment for cervical cancer. An international cancer registries study. Cancer 1995;76:442-52.

10. Curtis RE, Boice JD Jr., Stovall M, Bernstein L, Holowaty E, Karjalainen S, et al. Relationship of leukemia risk to radiation dose following cancer of the uterine corpus. J Natl Cancer Inst 1994;86:1315-24.

11. Hankey BF, Curtis RE, Naughton MD, Boice JD Jr., Flannery JT. A retrospective cohort analysis of second breast cancer risk for primary breast cancer patients with an assessment of the effect of radiation therapy. J Natl Cancer Inst 1983;70: 797-804.

12. Neugut AI, Ahsan H, Robinson E, Ennis RD. Bladder carcinoma and other second malignancies after radiotherapy for prostate carcinoma. Cancer 1997;79:1600-4.

13. Pawlish KS, Schottenfeld D, Severson R, Montie JE. Risk of multiple primary cancers in prostate cancer patients in the Detroit metropolitan area: a retrospective cohort study. Prostate 1997;33:75-86.

14. Boice JD Jr., Curtis RE, Kleinerman RA, Flannery JT, Fraumeni JF Jr. Multiple primary cancers in Connecticut, 193582. Yale J Biol Med 1986;59:533-45.

15. Ries LAG, Kosary CL, Hankey BF, Miller BA, Harras A, Edwards BK, editors. SEER Cancer Statistics Review 1973-1994. Bethesda (MD): National Institutes of Health, National Cancer Institute; 1997 DHHS Pub No. (NIH) 97-2789.

16. Mettlin CJ, Murphy GP, Sylvester J, McKee RF, Morrow M, Winchester DP. Results of hospital cancer registry surveys by the American College of Surgeons. Outcomes of prostate cancer treatment by radical prostatectomy. Cancer 1997;80: 1875-81. 
17. Breslow NE. The design and analysis of cohort studies Lyon: International Agency for Research on Cancer, 1987.

18. Mettlin CJ, Murphy GP, Rosenthal DS, Menck HR. The National Cancer Data Base report on prostate carcinoma after the peak in incidence rates in the U.S. The American College of Surgeons Commission on Cancer and the American Cancer Society. Cancer 1998;83:1679-84.

19. Kleinerman RA, Liebermann JV, Li FP. Second cancer following cancer of the male genital system in Connecticut, 1935-82. Monogr Natl Cancer Inst 1985;68:139-47.

20. Thompson DE, Mabuchi K, Ron E, Soda M, Tokunaga M, Ochikubo S, et al. Cancer incidence in atomic bomb survivors. Part II: solid tumors, 1958-1987. Radiat Res 1994;137(2 Suppl):S17-67.

21. Preston DL, Kusumi S, Tomonaga M, Izumi S, Ron E, Kuramoto A, et al. Cancer incidence in atomic bomb survivors. Part III. Leukemia, lymphoma and multiple myeloma, 1950-1987. Radiat Res 1994;137(2 Suppl):S68-97.

22. Mole RH, Papworth DG, Corp MJ. The dose-response for $\mathrm{x}$-ray induction of myeloid leukaemia in male CBA/H mice. Br J Cancer 1983;47:285-91.

23. Brenner DJ. The influence of cell killing on radiation oncogenesis: possible implications for high-LET risk assessment at medium doses. Health Phys 1993;65:358-66.

24. Boice JD Jr., Engholm G, Kleinerman RA, Blettner M, Stovall $\mathrm{M}$, Lisco H, et al. Radiation dose and second cancer risk in patients treated for cancer of the cervix. Radiat Res 1988; 116:3-55.

25. Kuttesch JF Jr., Wexler LH, Marcus RB, Fairclough D, Weaver-McClure L, White M, et al. Second malignancies after Ewing's sarcoma: radiation dose-dependency of secondary sarcomas. J Clin Oncol 1996;14:2818-25.

26. Wong FL, Boice JD Jr., Abramson DH, Tarone RE, Kleinerman RA, Stovall M, et al. Cancer incidence after retinoblastoma. Radiation dose and sarcoma risk. JAMA 1997;278: 1262-7.

27. Le Vu B, de Vathaire F, Shamsaldin A, Hawkins MM, Grimaud E, Hardiman C, et al. Radiation dose, chemotherapy and risk of osteosarcoma after solid tumours during childhood. Int J Cancer 1998;77:370-7.

28. Brown JM. The effect of acute $\mathrm{x}$-irradiation on the cell proliferation kinetics of induced carcinomas and their normal counterpart. Radiat Res 1970;43:627-53.

29. Leibel SA, Hanks GE, Kramer S. Patterns of care outcome studies: results of the national practice in adenocarcinoma of the prostate. Int J Radiat Oncol Biol Phys 1984;10:401-9.

30. Francois P, Beurtheret C, Dutreix A. Calculation of the dose delivered to organs outside the radiation beams. Med Phys 1988;15:879-83.

31. Khuri SF, Daley J, Henderson W, Barbour G, Lowry P, Irvin G, et al. The National Veterans Administration Surgical Risk Study: risk adjustment for the comparative assessment of the quality of surgical care. J Am Coll Surg 1995;180:519-31.

32. Rohan TE, Hislop TG, Howe GR, Gallagher RP, Teh CZ, Ghadirian P. Cigarette smoking and risk of prostate cancer: a population-based case-control study in Ontario and British Columbia, Canada. Eur J Cancer Prev 1997;6:382-8.

33. Boice JD Jr., Day NE, Andersen A, Brinton LA, Brown R, Choi NW, et al. Second cancers following radiation treatment for cervical cancer. An international collaboration among cancer registries. J Natl Cancer Inst 1985;74:955-75.

34. Storm H, Jensen OM. Second primary cancers among 40,518 women treated for cancer or carcinoma in situ of the cervix. In: Day NE, Boice JD Jr., editors. Second cancer in relation to radiation treatment for cervical cancer. Lyon: International Agency for Research on Cancer, 1983:59-69.

35. Stovall M, Blackwell CR, Cundiff J, Novack DH, Palta JR, Wagner LK, et al. Fetal dose from radiotherapy with photon beams: report of AAPM Radiation Therapy Committee Task Group No. 36. Med Phys 1995;22:63-82.

36. Zietman AL, Owen J, Mougham J, Katz A, Mahon I, Hanks GE. Radiation practice patterns for men with cancer of the prostate. The 1994 patterns of care study [abstract]. Int J Radiat Oncol Biol Phys 1998;42(Suppl 1):290.

37. Hall EJ, Martin SG, Amols HI, Hei TK. Photoneutrons from medical linear accelerators-radiobiological measurements and risk estimates. Int J Radiat Oncol Biol Phys 1995;33:22530 .

38. Zelefsky MJ, Leibel SA, Gaudin PB, Kutcher GJ, Fleshner NE, Venkatramen ES, et al. Dose escalation with three-dimensional conformal radiation therapy affects the outcome in prostate cancer. Int J Radiat Oncol Biol Phys 1998;41:491500.

39. Hanks GE, Hanlon AL, Pinover WH, Horwitz EM, Schultheiss TE. Survival advantage for prostate cancer patients treated with high-dose three-dimensional conformal radiotherapy. Cancer J Sci Am 1999;5:152-8. 